\title{
DIFFICULTIES IN THE ECHOCARDIOGRAPHIC DIAGNOSIS OF BLAND-WHITE-GARLAND SYNDROME
}

\author{
P. Shivachev, L. Marinov \\ Department of Pediatrics \\ Medical University "Prof. Dr Paraskev Stoyanov" - Varna, Bulgaria
}

\section{SUMMARY}

The Bland-White-Garland Syndrome (BWG) is a rare, but serious congenital cardiac anomaly, found in 1/300 000 life born children or is $0,25-0,5 \%$ of the congenital heart anomalies (CHA). At the same time it is the most frequent congenital coronary anomaly - $90 \%$. It is described for the first time in 1866, but the full clinical-anatomic description is given from Bland, White and Garland in 1933, and that is why it takes their names. It represents anomaly of the start of the left coronary artery from the trunk of the pulmonary artery (ALKAPA), most often from the left sinus with signs of myocardial ischemia in the relevant zones. In evolution develop collaterals between both coronary arteries and the syndrome of "stealing" because of the presence of significant left-right shunt on vascular level. Connected to this there are two forms of the disease: infantile and adult.

The aim of this report is to present the ECG and EchoCG results, clinical manifestation and result of the held conservative and operative treatment in three children, hospitalized in the clinic during the last years, establishing diagnostic difficulties in the echocardiograph examination and specifying of the type of the congenital cardiac malformation.

Key words: Bland-White-Garland syndrome, EchoCG, ECG, clinical manifestation, outcome of the disease.

The Bland-White-Garland Syndrome (BWG) is a rare, but serious congenital cardiac anomaly, found in 1/300 000 life born children or is $0,25-0,5 \%$ of the congenital cardiac anomalies (CCA). At the same time it is the most frequent congenital coronary anomaly - $90 \%$. It is described for the first time in 1866, but the full clinical-anatomic description is given from Bland, White and Garland in 1933, and that is why it takes their names. It represents anomaly of the start of the left coronary artery from the trunk of the pulmonary artery (ALCAPA), most often from the left sinus with signs of myocardial ischemia in the relevant zones. In evolution develop collaterals between both coronary arteries and the syndrome of "stealing" because of the presence of significant left-right shunt on vascular level. Connected to this there are two forms of the disease: infantile and adult. The anomaly most often if isolated, but it can be combined with other CCA - interventricular defects, persisting arterial channel, tetralogy of Fallot or aortic coarctation. The early diagnosis is often crucial for the prognosis and outcome of the operative treatment.

The aim of this report is to present three children hospitalized in the clinic, during the last years, which established diagnostic difficulties in the echocardiograph examination and specifying of the type of the congenital cardiac malformation.

Case 1. T. V. V.- 8 months. A female baby, from first normal pregnancy and birth, full term child with weight 2850 $\mathrm{g}$, smooth afterbirth period. After the third month is observed a lowering of the weight of the child. At the age of 7 months it was hospitalized in the pediatric department of Hospital Dobrich with symptoms of pneumonia, severe pulmonary and cardiac insufficiency, imposing apparatus ventilation, anemia, hypoglycemia, metabolite acidosis, highgrade cytolysis (suspicion of Rey syndrome), meningism, DIC syndrome and episodes of clinical death. The condition of the child improved after the use of Dobutamin. The patient is directed to the clinic with signs of salient cardiac insufficiency.

CVS - marked parasternal vosur, protodiastolic gallop rhythm, distanced I tone, protomesosystolic murmur $2+/ 6$ grade on the cardiac bases, propagating to the back. Developed hepatosplenomegaly with liver at $6 \mathrm{~cm}$, spleen at $2,5 \mathrm{~cm}$ under the costal line.

ECG - sinus tachycardia, horizontal heart position, ischemic type of the changes in the repolarization in I, aVL and left chest leads, signs of developed left atrial and left ventricular overload.

Chest X-ray - cranial deviation of the pulmonary stream, developed cardiomegaly.

Echocardiography - small left pleural effusion and small pericardial effusion. Developed dilatation of the left cardiac cavities. LV - 40/27 mm, hypocontractile with FS $30 \%$. "Sank" mitral valve with regurgitation to I grade. Dilateted RCA - $4 \mathrm{~mm}$. The anomalous start of LCA from the back-lateral sinus of the pulmonary artery (fig. 1.) is directly observed. Turbulent blood stream in PA. Lowered 
bloodstream in the ascendant and descendent aorta. The child is sent for operative treatment in PCC in NCCVD Sofia, where by intracardial examination the diagnose was proved, and a reimplantation of the ALCA to the aortic root was performed. After the operation the child is with normal cardiac status and physical development.

Case 2. V. V. H. - 5 months. A female baby, from I pregnancy with bleeding in $2 \mathrm{~m}$.1., born by Sectio Cezarea because of low birth activity, full term with weight $3200 \mathrm{~g}$. The child was hospitalized in the clinic at the age of 13 days, with signs of cardiac insufficiency. Membranous interventricular defect with elements of oblique channel, interatrial defect - foramen ovale and persistent arterial channel were found (fig. 2.). Cardiotonic and anticongestive treatment was started. The child was transferred to PCC of $\mathrm{NCH}$ - Sofia.

The main diagnose was proved with the presence if dilatation of the left cavities with diastolic size of LV - 24mm, slight depression of the pump function (EF - 50\%) and subsystemic pulmonary hypertension. In addition are found 3 small, muscular, channel defects, restrictive foramen ovale and closed arterial channel. The treatment of the cardiac insufficiency was continued. Through the next months the child was in damaged general condition with uncontrolled cardiac insufficiency and hypotrophy. At the age of 5 months during control hospitalization in Sofia was made the suggestion for BWG syndrome, proved by intracardial examination and an operative correction was performed. The post operative condition stayed damaged with uncontrolled cardiac insufficiency and hypotrophy. On echocardiography was found small pericardial effusion, marked dilatation of LV - 47/36,5 mm, with FS 22\% and EF - 53\% and extremely narrowed LCA - 1,2 mm. The child died at the age of 6 months in our clinic.

Case 3. T. J. Z. -2 months. A male baby from II normal pregnancy and birth, full term with weight $3750 \mathrm{~g}$. Familial anamnesis - grandfather with arrhythmia. At the age of 1 year and 18 days it was hospitalized in Hospital "St. Anna" - Varna, because of increased breathing, lowered appetite, no weight growth and bluing for a week. The child was transferred to our clinic with suspicion for acute myocarditis. It was admitted in damaged condition with signs of cardiac insufficiency. CVS - distanced heart tones, protomesosystolic murmur 2-3/6 grade in IV left intercostals space. Liver - at $3,5 \mathrm{~cm}$ under costal line.

ECG - sinus rhythm, vertical heart position, burdened left ventricle with deep Q- wave and ST-elevation in the left precordial leads.

Chest X-ray - slight cranial deviation of the pulmonary circulation, enlarged, mainly in left, cardiac shadow.

Echocardiography - restrictive left-right shunt through foramen ovale. Enlarged left cardiac cavities - 36/ $26 \mathrm{~mm}$ with lowered contractile indexes (FS 26\%, EF 52\%) and proportion LA/Ao - 2,0, MI - II grade and subsytemic pulmonary hypertension. In the bidimensional scan was found normal start of the LCA (fig. 3.). Cardiotonic and anticongestive treatment was started. A work diagnose DCMP was accepted and the child was directed for consultation in PCC of NCH - Sofia. The last one was not performed because of worsening of the condition of the child with increased decompensation symptoms, which led to new hospitalization in the clinic at the age of 2,5 months. After treatment the child was transferred in NCH - Sofia, where was found anomaly start of the LCA from PA. An operation was performed, but the end was with exitus at the age of three months.

\section{DISCUSSION:}

ALCAPA is a rare, but serious CHM, and most of the patients die at early infancy. The anomaly is tolerated in the fetus because of the similar pressures in aorta (Ao) and pulmonary artery (PA), nonrestrictive PAC and almost equal oxygenation in the parallel circulations. After birth, because of lowering of the pulmonary vascular resistance and the pressure in the PA, the myocardial zones, perfused by ALCA are put in regime of hypoperfusion and lowered oxygenation with development of myocardial ischemia and infarcts. The development of collateral net between both coronary arteries, leads to left-right shunt and "steal" syndrome. In approximately $85 \%$ of ALCAPA, the clinical manifestation is during the first 1-2 months of life, as the mortality rate during the 1 year is $90 \%$. Small part of the patients survives and reaches bigger ages - adult form (2). Their clinical manifestation is unspecific and most often must be differentiated between dilatative cardiomiopathy (DCM), coronary fistula, mitral insufficiency (MI), and virus miocarditis.

Nowadays the prognosis is improved due to the early diagnosis with the use of echocardiography (EchoCG) with color Doppler and due to the contemporary surgical techniques, including myocardial perfusion. Wall D. And coauthors, based on 29 years of trials, observed mortality in $18 \%$. Generally the postoperative distant prognosis is good with medial FS - 36,3\%/9,9\% and no MI in 80\% (7).

Most of the cases with ALCAPA can be diagnosed by echocardiography. With special attention must be observed infants with left ventricular dysfunction, abnormalities in the movements of the wall of the left ventricle and mitral insufficiency (MI). There is dilatation of RCA, which corresponds to the level of collateralization. Insensitive, but specific finding is the increased echogenity of the papillary muscles (PM). The direct visualization of the anomal start of the LCA of PA is possible, as it was in the first child that we observed. EchoCG can not be enough for the correct diagnosis. The anomaly coronary artery can 
cross near to the aortic sinus, causing impression and image of normal start of the LCA, as it was in the last observed child. Analyzing the abilities of the EchoCG in 8 patients with ALCAPA, Yang Ya-Li and coauthors, found that 4 of them were with mistaken diagnosis: 2 cases interpreted as fistula between RCA and PA and 2 - with normal LCA, because of visualization of linear structure, starting form the Ao (8). Karr SS and coauthors, find that by bidimensional EchoCG there are false results in 50\% - normal start of the LCA from Ao root. The use of colored Doppler significantly increases the diagnostic abilities of EchoCG by establishing of abnormal jet in the trunk of PA and of retrograde bloodstream in the system of LCA. The direction of the bloodstream can be described in 3 of 10 children with ALCAPA (5). In difference from PAC, the retrograde flow in the trunk of PA is with unusual orientation. According to Pisacane and coauthors, the visualization of intercoronary collaterals can direct to the diagnosis (6). In very early ages there can be no collaterals and respectively retrograde flow. Rarely ALCA can start from branches of the PA, which makes difficult the EchoCG diagnosis, even Doppler. In some cases, especially in the adult forms, the transesophageal EchoCg, can be more informative from transtoracle $(4,6)$. Chanq RR and Allada V. iInvestigate 23 patients with ALCAPA (middle age 6 months) and 23 with DCM (middle age 5,5 months) with the aim of setting of ECG and EchoCG criteria for differentiation of the cases with ALCAPA from those with DCM. The results are analyzed by logistic regression analysis and scoring system for setting of diagnose. For significant differentiation diagnostic features are determined: QT model in aVL with Q-wave $\geq 3 \mathrm{MM}$, with

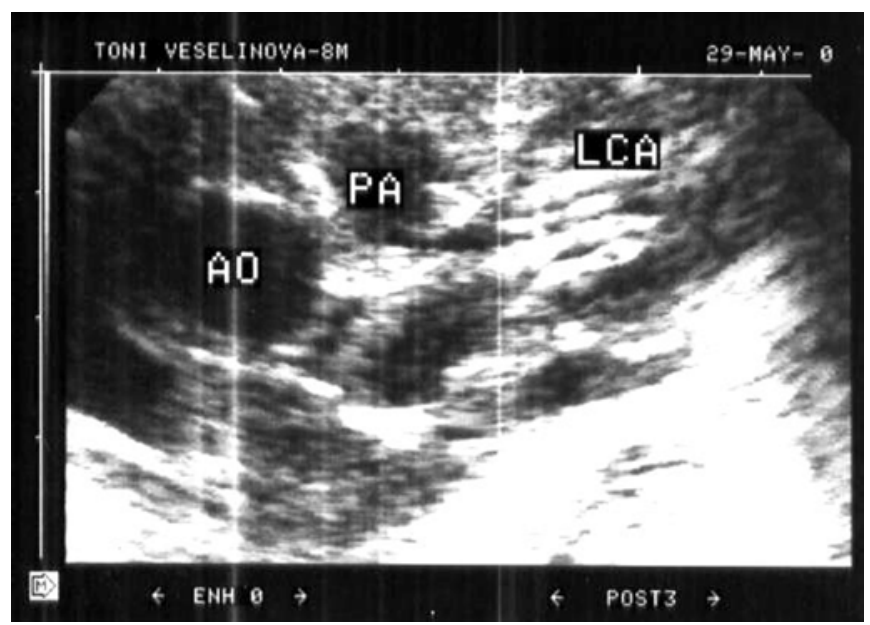

Fig. 1. Abnormal outcome of LCA out of posteriorlateral sinus of PA invert T-waves on ECG, RCA at Ao root $\geq 0,14$, higher echogenity of PM, color Doppler in LCA from Ao or PA. Each of the indexes gives 1 point. The scoring system is with $100 \%$ sensitivity and specificity $91 \%$ for ALCAPA and is recommended for children with acute congestive cardiac insufficiency (1).

Even rarely ALCAPA can be combined with other CHM. In these cases is necessary special attention especially when the severity of the clinical manifestation does not correspond to the found cardiac anomaly. (case 3).

The intracardial examination and coronarography must be performed after negative EchoCg and suspicious clinical and ECG data for Bland-With-Garland syndrome. The injection of contrast material in the Ao root or selectively in RCA, fills retrograde LCA, and in some cases in the trunk of PA. in the lack of collaterals between Ao and RCA the arteriographies are uninformative. In these cases is necessary Stop flow angiography from PA for the imaging of ALCA.

\section{CONCLUSION:}

EchoCG is a diagnostic method, on first choice, when you suspect ALCAPA. Bidimensional EchoCG is with insufficient informative value. The use of the complex of modern technologies of the method is necessary, including color Doppler, as well as detailed knowledge of the anomaly for the setting of the correct diagnosis. In some of the cases is necessary the performing of aorto- and coronarographia. The early diagnosis and modern operative techniques are in the bases of improved prognosis of this disease.

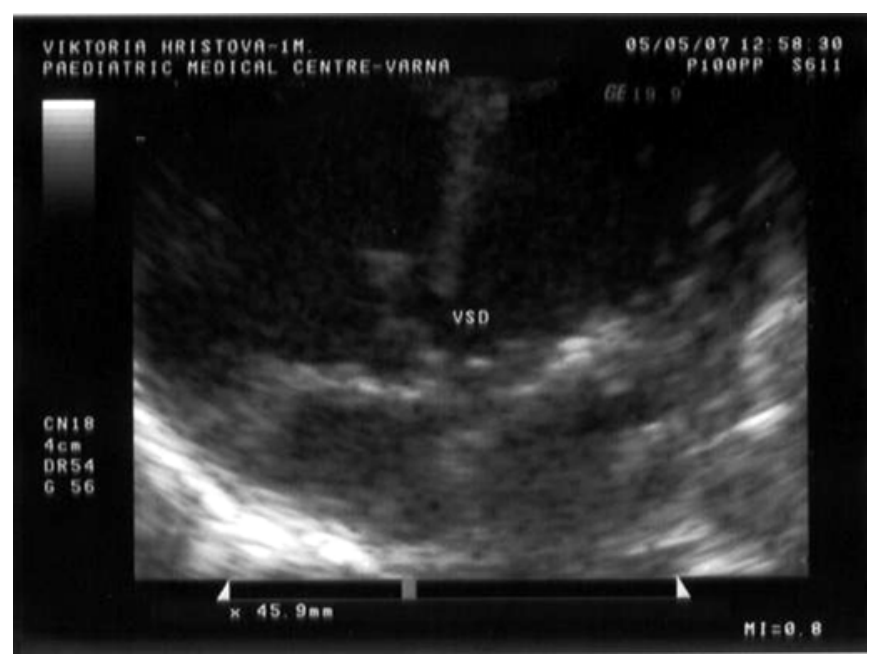

Fig. 2. Membraneous VSD with oblique canal elements 


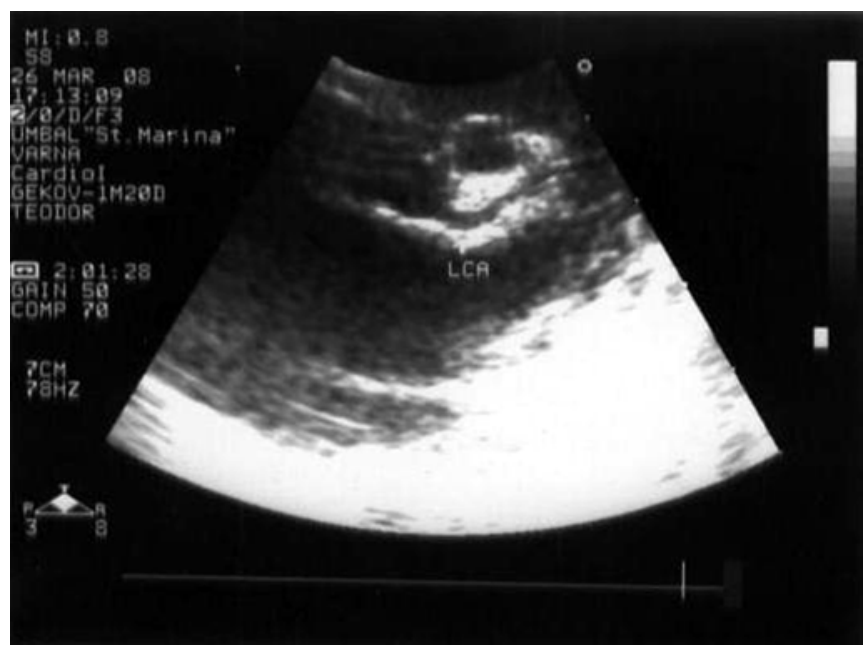

Fig. 3. Apparently normal outcome of LCA

\section{BIBLIOGRAPHY:}

1. Chanq R. R., Allada V. Electrocardiographic and echocardiographic features that distinguish anomalous origin of the left coronary artery from pulmonary artery from idiopathic dilated cardiomyopathi. Pediatr Cardiol. 2001 Jan-Feb; 22(1):3-10.

2. Drighil A., Chraibi S., Bennis A. Adult type anomalous origin of the left coronary artery from the pulmonary artery: When should we be awere? International Journal of Cardiology, Volume 113 (3). 2006: E119-E121.

3. Karr S. S., Parness I. A., Spevak P. J., et al. Diagnosis of anomalous left coronary artery by Doppler color flow mapping: distinction from other causes of dilated cardiomyopathy. J Am Coll
Cardiol. 1992. 19(6):1271-75.

4. Kececioglu D., Kotthoff S., Konertz W., et al. Pulmonary artery origing of the left coronary artery: diagnosis by transoesophagial echocardiography in infancy. European Heart Journal 1993 14(7):1006-1007.

5. Pisacane C., Pinto S. C., De Gregorio P., et al. "Steal" collaterals: an echocardiographic diagnostic marker for anomalous origin of the left main coronary artery from the pulmonary artery in the adult. J Am Soc Echocardiogr. 2006;19(1):107.e3107.e6.

6. Shun-Yi Hsu, Fun-Chung Lin, HernJia Chang, San-Jou Yeh and Delon Wu. Multiplane Transesophageal Echocardio- graphy in Diagnosis of Anomalous Origin of the Left Coronary Artery from the Pulmonary Atrery: A Case Report. Journal of the American Sosiety of Echcardiography, 1998. 11 (6): 668-672.

7. Wall D., Shehatha J., Konstantinov I., et al.Homian Jalali and Peter Pohlmer. ALCAPA ( Anomalous origing of the left coronary artery from the pulmonary artery) the 29 year Prince Charles Hospital experience. Heart, Lung and Circulation. 2007; 16, Suppl. 1: S43.

8. Yang Y. L., Nanda N. C., Wang X. F. et al. Echocardiographic Diagnosis of Anomalous Origing of the Left Coronary Artery from the Pulmonary Artery. Echcardiography, 2007. 24 (4): 405-411.

\section{Corresponding Author:}

Lachezar Marinov MD, $\mathrm{PhD}$

Department of Pediatrics

Medical University "Prof. Dr Paraskev Stoyanov"

55 Marin Drinov Str., 9000 Varna, Bulgaria

Tel.: +359/899 670525 ;

E-mail:marrinovs@abv.bg 